Urologe $2017 \cdot 56: 449-450$

DOI 10.1007/s00120-017-0344-1

Online publiziert: 28. Februar 2017

(c) Springer Medizin Verlag GmbH 2017

CrossMark

\author{
Marc-Oliver Grimm ${ }^{2}$ - Gerd Lümmen ${ }^{1}$ \\ 'Urologische Abteilung, St. Josef Hospital, Troisdorf, Deutschland \\ ${ }^{2}$ Klinik und Poliklinik für Urologie, Universitätsklinikum Jena, Jena, Deutschland
}

\title{
Nebenwirkungen medikamentöser Therapie in der Urologie
}

Ein nicht unerheblicher Teil urologischer Therapiemöglichkeiten besteht im Einsatz unterschiedlicher Medikamente in verschiedenen Indikationsbereichen. Neben der richtigen Indikationsstellung stellen insbesondere die Kenntnisse der Nebenwirkungen und deren Management einen wichtigen Pfeiler innerhalb der konservativen Therapie in der Urologie dar. Um Ihnen hier einen umfassenden Überblick über potenzielle Nebenwirkungen zu geben, haben wir in den jeweiligen Indikationsbereichen namhafte Autoren gewinnen können, die die jeweiligen Substanzklassen bzw. Medikamente beleuchten.

Der erste Beitrag behandelt das $\mathrm{Ne}$ benwirkungsspektrum der medikamentösen erektilen Dysfunktionsbehandlung. Die weitverbreiteten Phoshodisesterase-5-Inhibitoren sind bei gleichzeitiger Einnahme von Nitraten sowie bei Vorliegen einer Retinitis pigmentosa wie auch bei der NAION (nicht-arteriitische anteriore ischämische Optikusneuropathie) kontraindiziert. Zu denken ist aber auch an den Priapismus unter intrakavernöser Therapie mit Prostaglandinen oder Papaverin.

Der zweite Beitrag befasst sich mit den Nebenwirkungen der medikamentösen Therapie der Blasenspeicher- und Entleerungsstörungen und gibt einen Überblick von a1-Blockern über Antimuskarinika, 5a-Reduktaseinhibitoren, Phosphodiesterase-5-Inhibitoren bis hin $\mathrm{zu}$ $\beta 3$-Antagonisten. Neben den insgesamt als gut verträglich geltenden $\alpha 1$-Blockern sind bei den anderen Substanzen deutlich mehr Nebenwirkungen zu berücksichti- gen, bei z. T. noch fehlenden Langzeitdaten.

Der nächste Themenblock befasst sich mit der umfangreichen und häufig durchgeführten antihormonellen Therapie beim Prostatakarzinom und deren Nebenwirkungsprofil. Unter der sog. Androgenentzugstherapie kommt es zu Nebenwirkungen auf verschiedenen Stoffwechselebenen sowie kardiovaskulären Komplikationen und Auswirkungen auf die Psyche der Patienten. Zusätzlich zu dem Nebenwirkungsspektrum wird von den Autoren auch die Entwicklung von Resistenzen gegenüber den primären und sekundären Hormontherapien beleuchtet.

Der nachfolgende Beitrag berichtet über die potenziellen Nebenwirkungen der Chemotherapeutika in der Urologie und deren Management. Beginnend mit der asymptomatischen und symptomatischen Neutropenie über die chemotherapieinduzierte Nausea und Emesis, Stomatitis, Mukositis und Diarrhö, stellt der Beitrag die klassischen chemotherapiebedingten Nebenwirkungen in umfangreicher Weise dar.

In einem weiteren Beitrag werden die Nebenwirkungen der Schmerztherapie, suffiziente Analgesie mit vermeidbaren Komplikationen dargestellt. Neben der Differentialindikation sind Kenntnisse der Nebenwirkungen angewendeter Verfahren Voraussetzung einer erfolgreichen Schmerztherapie. Dabei werden wesentliche Verfahren der Schmerztherapie mit ihren spezifischen Nebenwirkungen vorgestellt, die in Kombination gegebenenfalls additiv wirken können. Für eine effektive und tolerable Analgesie müssen die Risiken individuell abgewogen und mit dem Patienten besprochen werden. Hierzu ist ein umfangreiches Wissen erforderlich.

Der letzte Beitrag befasst sich mit den Nebenwirkungen der neu eingeführten Checkpoint-Inhibitoren. Diese, das Immunsystem modulierenden Substanzen, sind insgesamt gut verträglich, zeigen allerdings gelegentlich sog. immunvermittelte Nebenwirkungen. Hierzu zählen Hautausschläge, Kolitis, Hepatitis, Pneumonitis, Nephritis und Endokrinopathien bis hin zur Hypohysitis. Kenntnisse des Nebenwirkungsspektrums dieser Substanzen, der entsprechenden (Differential-)Diagnostik und des Therapiemanagements sind für eine sichere Anwendung dieser Substanzen entscheidend.

Ziel der Beiträge in diesem Heft ist es, Ihnen durch die zusammenfassende Darstellung potentieller Nebenwirkungen eine Hilfestellung für den klinischen Alltag zu liefern. Nur mit dem Wissen der Wirkungen und Nebenwirkungen der verschiedenen Medikamente können ggf. Überdosierungen und Komplikationen vermieden werden.

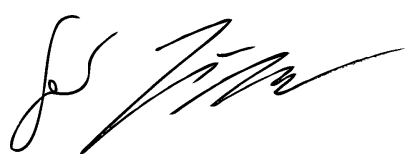

Gerd Lümmen

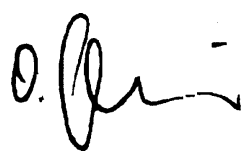

Oliver Grimm 


\section{Korrespondenzadresse}

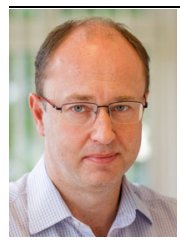

Prof. Dr. M.-0. Grimm

Klinik und Poliklinik für Uro-

logie, Universitätsklinikum

Jena

Lessingstraße 1, 07743 Jena,

Deutschland

Marc-Oliver.Grimm@

med.uni-jena.de

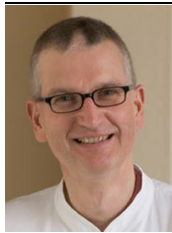

Prof. Dr. G. Lümmen

Urologische Abteilung, St.

Josef Hospital

Hospitalstr. 45, 53840 Trois-

dorf, Deutschland

gerd.luemmen@

josef-hospital.de
Interessenkonflikt. G. Lümmen und gibt an, dass

kein Interessenkonflikt besteht. M.-0. Grimm: For-

schungsunterstützung von Novartis und Bristol-Myers

Squibb, Beratungs- und/oder Referentenhonorare:

Amgen, AstraZeneca, Astellas, Bayer Health Care, Bris-

tol-Myers Squibb, GlaxoSmithKline, Hexal, Janssen

Cilag, JenaPharm, Novartis, Pfizer, Perre Fabre, Sanofi,

and Teva.

\section{e.Med Gynäkologie \& Urologie Das Fortbildungs-Abo passend zu Ihrem Fachgebiet}

SpringerMedizin.de bietet allen Ärzten, die sich effizient auf dem Laufenden halten wollen, ein maßgeschneidertes Fortbildungs-Abo mit CME-Fortbildungen und Premium-Inhalten der Fachzeitschriften passend zu Ihrem Fachgebiet. Inklusive einer gedruckten Zeitschrift nach Wahl.

\section{$>$ Gezielt recherchieren, schnell und aktuell informieren: Mit e.Med Gynäkologie \& Urologie erhalten Sie Zugriff auf die gynäkologischen und urologischen Fachzeitschriften und die dazugehörigen Fortbildungen.}

$>N$ CME-Punkte zu sammeln.

> Profitieren Sie von der aktuellen und fundierten Berichterstattung über Entwicklungen und Neuheiten in der Gynäkologie und Urologie.
Greifen Sie auf die Volltexte von deutschen und englischsprachigen gynäkologischen und urologischen Fachzeitschriften zu - direkt auf der Website oder als PDF-Download.

Darüber hinaus ist im e.Med Gynäkologie \& Urologie Abo eine gynäkologische oder urologische Springer Medizin Fachzeitschrift Ihrer Wahl enthalten, die Ihnen regelmäßig per Post zugesandt wird.

Testen Sie e.Med Gynäkologie \& Urologie 30 Tage kostenlos und unverbindlich!

Jetzt informieren unter

http://bit.ly/2ieYqHD

oder telefonisch unter 0800-77 80777

(Montag bis Freitag, 10 bis $17 \mathrm{Uhr}$ ) 